\title{
$\frac{5}{3}$ Digital Publisher ISSN 2588-0705 \\ Revista digital de investigación \\ Volumen 5 Edición especial

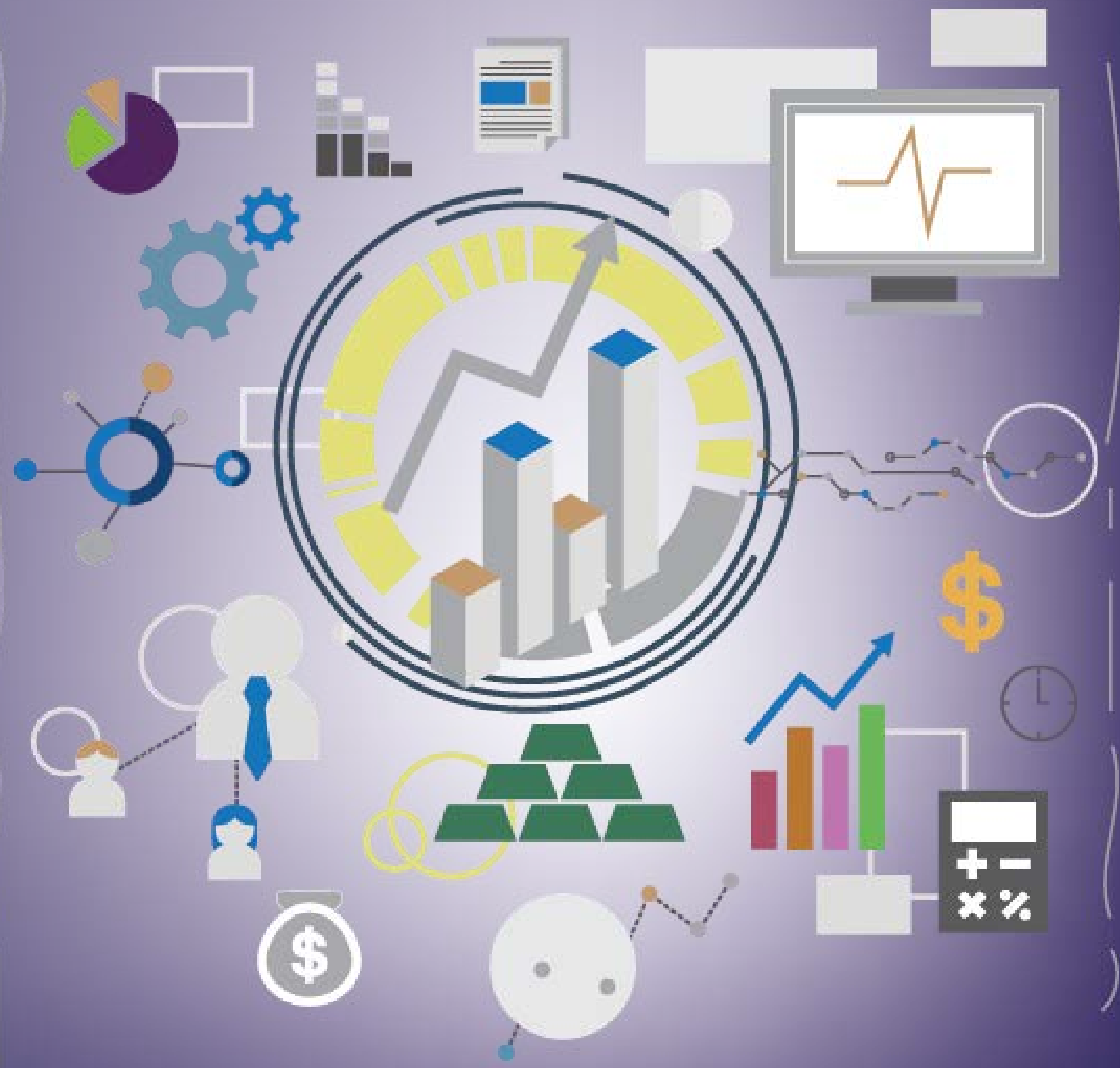

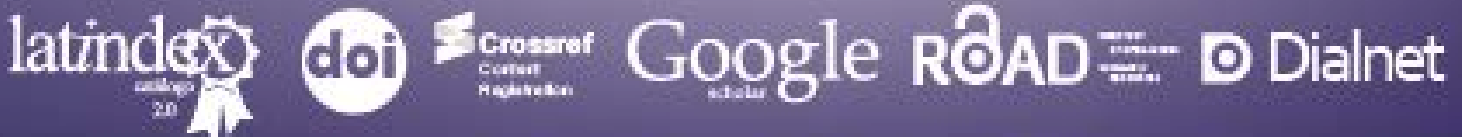




\title{
昜Digital Publisher
}

ISSN 2588-0705

\section{Impuesto a las ganancias, conciliación tributaria entre NIIF y la LORTI}

\author{
Income tax, tax reconciliation \\ between IFRS and LORTI
}

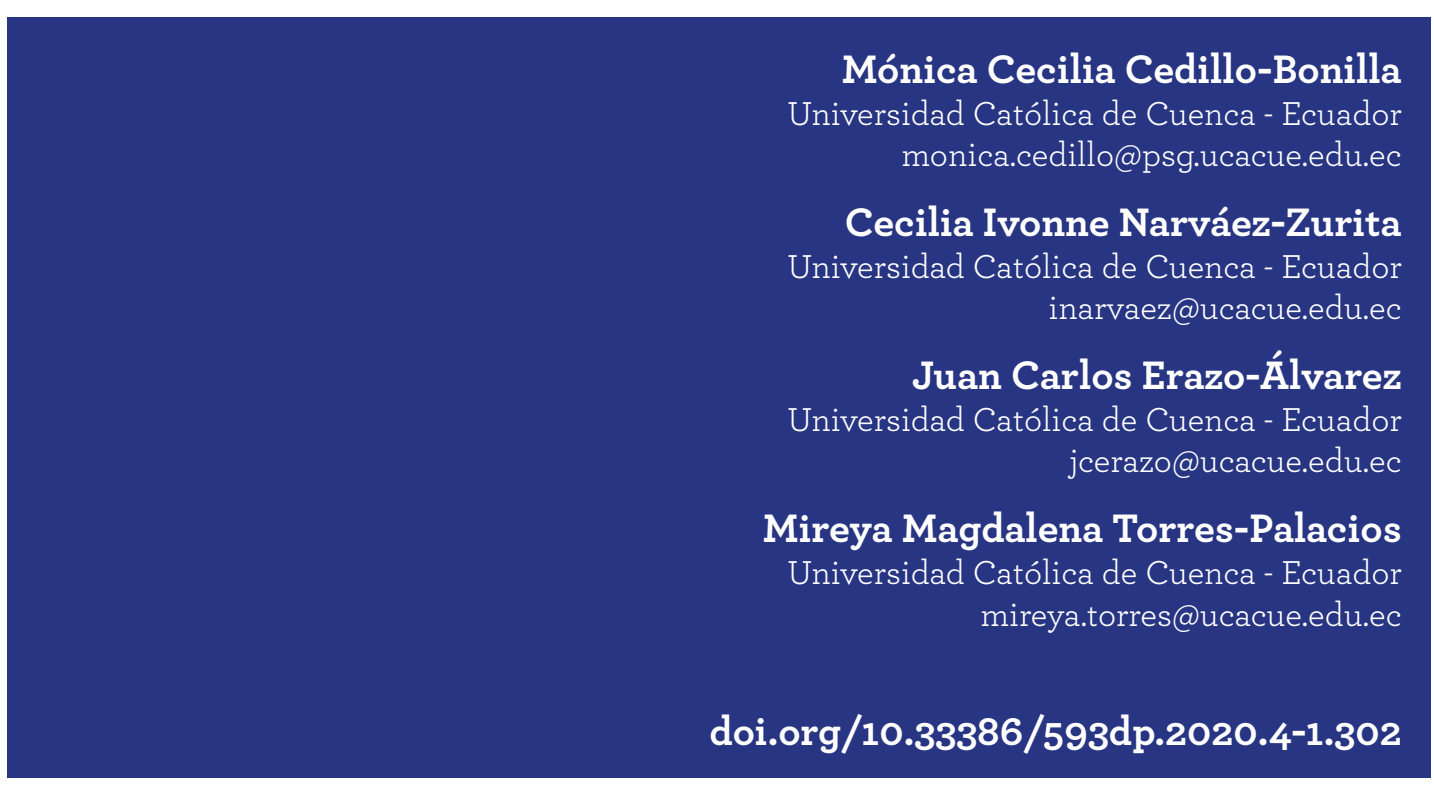

V5-N4-1 (ago) 2020, pp. 137-152 | Recibido: 30 de julio de 2020 - Aceptado: 15 de julio de 2020 (1 ronda rev.) 


\section{RESUMEN}

La proliferación de empresas internacionales y la globalización de la economía, ha hecho necesario el desarrollo de normativas estandarizadas para el manejo de la contabilidad en el mundo. Entre ellas están las normas internacionales de información financiera (NIIF) y las normas internacionales de contabilidad (NIC), con el fin de fomentar negociaciones e inversiones internacionales. El Ecuador entró en proceso de adopción de las NIIF a partir del año 2010 bajo el control de la Superintendencia de Compañías, Valores y Seguros, dicha conversión produjo varios inconvenientes, entre ellos, la conciliación tributaria, dando origen a las diferencias temporarias y a los impuestos diferidos. El objetivo del presente estudio es diseñar un procedimiento metodológico para la aplicación de la NIC 12 en la Agencia de Publicidad Ad/a, haciendo factible el análisis de las diferencias que surgen en la conciliación contable y fiscal. La investigación tuvo un enfoque cuantitativo - cualitativo con un alcance descriptivo - explicativo - transaccional. En la fase de diagnóstico se detectó que la empresa, no aplica los impuestos diferidos, afectando al estado de resultados y situación financiera. Adicional se recalca la importancia de capacitar al personal, en el tratamiento del impuesto diferido, generado a partir de las diferencias temporarias.

Palabras clave: impuesto a las ganancias, conciliación tributaria, activos por impuestos diferidos, pasivos por impuestos diferidos, diferencias temporarias.

\section{ABSTRACT}

Cómo citar este artículo:

APA

Cedillo, M. (2020). Impuesto a las ganancias, conciliación tributaria entre NIIF y la LORTI . 593 Digital Publisher CEIT, 5(4-1), 137-152. https://doi. org/10.33386/593dp.2020.4-1.302

Descargar para Mendeley Zotero

The proliferation of international companies and the globalization of the economy has made it necessary to make standardized regulations for accounting management. These include international financial reporting standards (IFRS) and international accounting standards (IAS), in order to encourage international negotiations and investments. Ecuador entered the process of adopting IFRS since 2010, the process was controlled by the Superintendency of Companies, Securities and Insurance, and brought several problems, including tax conciliation, giving rise to temporary differences and taxes deferred. The objective of this study is to carry out a methodological procedure for the application of IAS 12 in the Company Ad / a, making possible the analysis of the differences that arise in the accounting and fiscal reconciliation. The research had a quantitative - qualitative approach with a descriptive - explanatory - transactional scope. It was detected that the company does not apply deferred taxes, affecting the income statement and financial situation. Additionally, the study detected the importance of training employees in the treatment of deferred tax, generated from temporary differences.

Keywords: income tax, tax reconciliation, deferred tax assets, deferred tax liabilities, temporary differences. 


\section{Introducción}

La globalización de la economía y la apertura de los mercados internacionales, ha hecho posible que profesionales contables generen normativas y estándares para facilitar a empresas de todo el mundo la comunicación de sus estados financieros, volviéndolas más cotizadas y competitivas en mercados internacionales, al facilitar su análisis mediante herramientas estratégicas como son las Normas Internaciones de Información Financiera (NIIF), que ponen a las empresas al mismo nivel de sus pares internacionales permitiendo su comparabilidad, además de brindarles ventajas internas, como balances y estados de resultados transparentes y confiables, con los que será posible una toma de decisiones más acertada para sus directivos .

Las Normas Internacionales de Información Financiera (NIIF) tienen gran aceptación en países alrededor del mundo, pues su uso permite a la empresa estar equiparada a nivel internacional y subir su valor. La Agencia de Publicidad Ad/a, dedicada al rubro de la publicidad por más de 10 años, con sede en la ciudad de Cuenca, Ecuador, tiene al menos treinta empleados de planta y factura anualmente alrededor de cuatro millones de dólares, por tanto, está obligada a presentar sus balances financieros de acuerdo a las NIIF, y al mismo tiempo, contribuir al Estado, de acuerdo a la Ley Orgánica de Régimen Tributario Interno (LORTI). El departamento contable de la empresa Ad/a, no tiene conocimiento sobre las normativas contables que deben aplicarse a los impuestos diferidos ni como se han de conciliar con las normativas tributarias. Esto constituye una perdida para la empresa, en cuanto existen valores que se pueden recuperar en ejercicios económicos futuros. Esta aplicación a medias de la normativa contable en mención, ha generado una tensión entre directivos y miembros del departamento contable, pues, por temor a multas por parte del Servicio de Rentas Internas (SRI), el área contable prefieren inclinarse al cumplimiento de las normas tributarias, dejando de lado el equilibrio que se debe lograr para sacar provecho de la recuperación de ciertos rubros, todo esto dentro de la ley que el mismo estado ecuatoriano garantiza.

En el Ecuador, la implementación de las Normas Internacionales de Información Financiera (NIIF) se dio en el año 2010, bajo orden y regulación de la Superintendencia de Compañías, Valores y Seguros (SUPERCIAS). La transición generó incomodidad en las empresas, dado el mayor número de exigencias, reportes e informes, en comparación con las NEC (normas ecuatorianas de contabilidad). A este hecho se suma la falta de concordancia entre NIIF y LORTI, ya que las NIIF desean obtener información financiera fiable, mientras que la normativa tributaria busca combatir la evasión de impuestos con la aplicación de la ley vigente en Ecuador (Cabrera, Narváez, \& Erazo, 2020).

En este contexto, la problemática de la investigación busca responder a la siguiente interrogante ¿cómo incide la aplicación de la NIC 12, en el cálculo del impuesto a la renta y en el proceso contable de la empresa Ad/a? tomando en cuenta que el cálculo del impuesto a las ganancias se trata de modo diferente desde el punto de vista contable que desde el tributario.

El objetivo de la presente investigación consiste en diseñar un procedimiento metodológico para la aplicación de la NIC 12 que permita la determinación de las diferencias que surgen en la conciliación contable y fiscal del objeto de estudio. Por un lado, se basa en los impuestos diferidos con la aplicación del método del pasivo basado en el balance, determinando así el impuesto a las ganancias, en el ejercicio de la Agencia de Publicidad Ad/a. Por otro lado, se analiza la normativa tributaria, cuya jurisdicción está por encima de las NIIF, por orden y decreto de los órganos rectores ecuatorianos.

Normas Internaciones de Información Financiera en el Ecuador

En los años setenta, profesionales contables de las más importantes potencias mundiales de la época, crearon el International Accounting Standards Committe (IASC), favoreciendo la 
estandarización de la información financiera con la publicación de más de 40 normas contables que sentaron la base para lo que en el año 2000, constituyó un nuevo organismo (Panchi, 2017). Alrededor de 100 países aportaron con más de 140 profesionales para conformar la International Accounting Standards Board (IASB), conocida en español como la Junta de Normas Internacionales de Contabilidad, sustituyendo así IASC o Comité de Normas Internacionales de Contabilidad.

El IASB ha publicado a la fecha, 13 Normas Internacionales de Información Financiera (NIIF) que fueron adoptadas en países de la Unión Europea, Latinoamérica y Asia. En Estados Unidos la normativa contable obligatoria es la US Generally Accepted Accounting Principles (US GAAP), sin embargo, las empresas estadounidenses con operaciones internacionales hacen uso de las dos normativas.

Otro reto para la IASB, es China, cuya resistencia ha cedido, por lo que el sitio oficial del organismo informa un plan de conversión para el año 2030 (OICE- Organización Interamericana de Ciencias Económicas, 2019). La figura 1 refleja la jurisdicción de las normativas impartidas por la IFRS

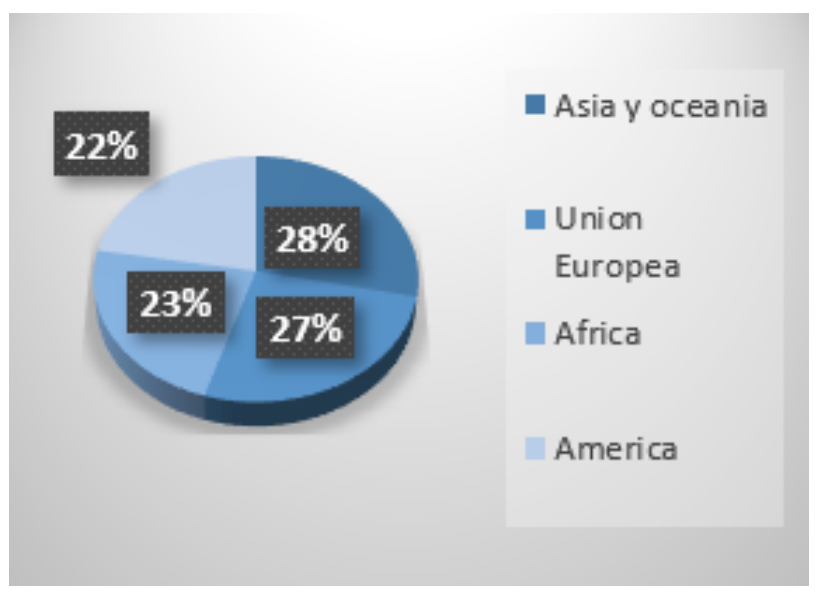

Figura 1: Jurisdicción de la IFRS en el mundo.

Fuente: (Instituto Mexicano de Contadores Públicos, 2015)

Todas las NIIF, tienen un propósito y están conformadas por varios criterios, en función de los cuales se preparan estados financieros de óptima calidad para el análisis de cualquier inversionista o directivo de una empresa. Su correcta aplicación está supeditada a la aplicación de reglas, principios y requieren un criterio profesional que las aplique de manera correcta, permitiendo generar estándares contables de alta calidad.

En el Ecuador, en el año 2010 a través de la superintendencia de Compañías, Valores y Seguros (SUPERCIAS) se declaró la obligatoriedad de la implementación de las NIIF para todas las empresas bajo control de la institución rectora en mención.

El proceso de adopción se dio en tres etapas, iniciando con la capacitación del personal a cargo, siguiendo con la evaluación del impacto que tendrá el proceso de transformación de NEC a NIIF y finalmente su aplicación a los procesos de alcance. La transición se llevó a cabo desde el año 2010, hasta 2012 bajo el cronograma contenido en la figura 2. (Superintendencia de Compañias, 2006).

\begin{tabular}{|l|l|}
\hline Año & \multicolumn{1}{|c|}{ Empresa } \\
\hline \multirow{2}{*}{2010} & $\begin{array}{l}\text { Ejercen actividad de auditoría } \\
\text { externa. }\end{array}$ \\
\cline { 2 - 2 } & $\begin{array}{l}\text { Sujetas por la ley de Mercado } \\
\text { de Valores. }\end{array}$ \\
\hline \multirow{2}{*}{2011} & $\begin{array}{l}\text { Poseen activos totales o iguales } \\
\text { a } \$ 4.000 .000,00 \text { al 31/12/2017. } \\
\text { Econom ía Mixta. }\end{array}$ \\
\cline { 2 - 2 } & $\begin{array}{l}\text { Holding tenedoras de acciones. } \\
\text { Sucursales de compañ ías } \\
\text { extranjeras que ejerzan } \\
\text { actividades en el Ecuador. } \\
\text { Las demás que no constan } \\
\text { en los grupos anteriores. }\end{array}$ \\
\hline
\end{tabular}

Figura 2. Implementación NIIF en el Ecuador.

Fuente: Superintendencia de Compañías del Ecuador. 
Las empresas dieron cuenta de múltiples inconvenientes, pues la implementación de las normas tenía que hacerse a los estados financieros del ejercicio fiscal de los años 2009, 2010 y 2011, detallando los ajustes y conciliaciones realizadas en el balance de la manera más minuciosa posible, aplicando la partida doble, en idioma español y en moneda de curso legal (Freire, 2010).

\section{NIIF para pymes en Ecuador}

La Superintendencia de Compañías, Valores y Seguros (SUPERCIAS), emitio una resolución mediante la cual, las empresas con activos menores a cuatro millones de dólares y que tengan menos de 200 trabajadores, podrían acogerse a la implementación de las NIIF para PYMES, tratamiento especial que también se ha dado en más de sesenta países alrededor del mundo, con una reducción bastante considerable de la normativa regular. Las NIIF completas en su versión 2010, tienen 3055 páginas y 3000 ítems en la hoja de verificación de cumplimiento, mientras que, las NIIF para PYMES en su versión 2009, tienen 370 páginas y 300 items por verificar. Los estados financieros para las PYMES comprenden: 1) estado de situación financiera, 2) estado de resultado 31 cambio de patrimonio, 4) flujo de efectivo, 5) Notas a los estados financieros (Solar \& Hidalgo, 20111 .

A la par del proceso de implementación de las NIIF y NIC, las empresas ecuatorianas deben cumplir con la Ley Orgánica de Régimen Tributario (LORTI), apareciendo la convergencia entre lo contable y lo tributario. La LORTI establece que llegado el caso de discrepancia entre las normativas contables versus normativas tributarias, siempre se dará prioridad a las tributarias (Juca, 2019).

\section{Normativa NIC 12}

En el año 2001 la IASB emitió la NIC 12: Impuesto a las Ganancias, su objetivo es la determinación del tratamiento contable del impuesto a la renta, considerando que, de las diferencias entre las declaraciones tributarias generadas a partir de la base fiscal y las declaraciones generadas a partir de la base contable, nacen los impuestos diferidos, los cuales deben ser asentados como activos o pasivos (Castro, 2016).

La NIC 12 tiene como objetivo fundamental "prescribir el tratamiento contable del impuesto a las ganancias" (IFRS Foundation @, 2019 , pág. A 1 129), sin embargo, el cumplimiento de la premisa genera inconvenientes derivados de buscar la recuperación futura de un activo y “... las transacciones y otros sucesos del periodo corriente que han sido objeto de reconocimiento en los estados financieros" (IFRS Foundation®, 2019 , pág. A 1 129).

La NIC 12 establece como obligatoriedad para las empresas llevar un registro contable de las consecuencias fiscales de transacciones y otros sucesos económicos. También, obliga a que los efectos fiscales de las transacciones sean llevados al patrimonio de forma directa.

Tiene incidencia sobre la plusvalía o cualquier otro reconocimiento de compra que puedan ser ventajosas para la empresa, exigiendo su reconocimiento como activos o pasivos por impuestos diferidos. Por otra parte, también realiza el reconocimiento de los activos por impuestos diferidos a perdidas y créditos fiscales no utilizados, presentando el impuesto a la renta con la inclusión de la información revelada de estos últimos (IFRS Foundation $®$, 2019).

La NIC 12 tiene alcance a todos los impuestos nacionales o extranjeros con relación a las ganancias obligadas a imposición y en cuanto a las conciliaciones exige "se tome una o ambas: Una conciliación de cifras que representan el gasto (ingreso) por impuestos y el resultado de multiplicar la ganancia contable por la tasa o tasas impositivas aplicables, o una conciliación numérica de los importes representativos de la tasa impositiva promedio efectiva y la tasa impositiva existente." (Hansen-Holm M. , 2011 ).

Otras exigencias considerables de la NIC 12 son expuestas en la figura 3. 


\section{Exigencia

$\begin{array}{lrl}\text { No reconocer } & \begin{array}{l}\text { Cuando surgen } \\ \text { pasivos por } \\ \text { impuestos diferidos. }\end{array} & \begin{array}{l}\text { reconocimiento } \\ \text { inicial de la } \\ \text { plusvalia. }\end{array}\end{array}$

\begin{tabular}{|c|c|}
\hline $\begin{array}{ll}\text { econoce un pasivo } \\
\text { or } \\
\text { ferido. }\end{array}$ & $\begin{array}{l}\text { Resultar del } \\
\text { reconocimiento } \\
\text { inicial de la } \\
\text { plusvalia. }\end{array}$ \\
\hline
\end{tabular} \\ Medir los activos o Medición en base pasivos por a consecuencias impuestos diferidos. fiscales derivadas del pago de activos o pasivos en libros.}

Figura 3. Exigencia y condiciones de la NIC 12. Fuente: (IFRS Foundation, 2013)

Cuando una empresa registra ganancias, la NIC 12 reconoce activos por impuesto diferidos de periodos anteriores, si, por el contrario ha registrado pérdidas y posee diferencias temporarias imponibles o deducibles de impuestos, podrá registrar activos por impuestos diferidos.

La norma tiene alcance para ser aplicada en la contabilización del impuesto sobre las ganancias y su aplicación dentro de la empresa debe ser evaluada cada año.

\section{Base Fiscal}

La Base Fiscal, está definida como las ganancias o pérdidas generadas dentro de un periodo tributario determinado. Se considera como la base fiscal de un activo, al valor pagado por éste y que es factible de deducción en el futuro, mientras que, la del pasivo es su valor luego de establecer una diferencia entre el mismo y sus propios gastos deducibles en periodos tributarios venideros (IFRS Foundation $₫, 2019$ ).

Los pasos a seguir para la determinación de la base fiscal se encuentran en la normativa NIC 12 y se exponen en la figura 4.
Determinacion de base fiscal

Rubro (Activos - Pasivos)

Si Activos = deducción

No activos = valor contable

Si pasivos = Valor contable ingreso exento futuro.

No Pasivos = valor contable deducción futura

Figura 4: Determinación de la Base Fiscal.

Fuente: (IFRS Foundation $囚, 2019$ )

El impuesto diferido

"Los impuestos diferidos son activos o pasivos creados a partir de una diferencia temporaria entre el resultado financiero y tributario de un periodo fiscal" (Instituto Mexicano de Contadores Públicos, 2015). Entre sus características están: al es un activo o pasivo no corriente, b) afecta a la utilidad y patrimonio, c) puede calcularse en el balance.

Para el cumplimiento de las exigencias planteadas por la NIC 12, en cuanto a los impuestos diferidos, ésta propone tres conceptos importantes:

a) Las diferencias temporarias son aquellos valores que constan entre el importe en libros de un activo o pasivo en el estado de situación financiera y su base fiscal, estas pueden ser: diferencias temporarias imponibles pues dan lugar a cantidades imponibles factibles de recuperación o liquidación (Omeñaca, 2009). 
Por otro lado, las diferencias temporarias deducibles, son como su nombre lo indica cantidades deducibles al determinar la ganancia o pérdida fiscal correspondiente a períodos futuros, cuando el importe en libros del activo es recuperado o el pasivo sea liquidado (Gay \& Amat, 2008). Las diferencias temporarias pueden clasificarse en imponibles y deducibles. Las diferencias temporarias imponibles generan un pasivo por impuesto diferido, y por consiguiente, se debe tributar. En tanto que, las diferencias temporarias deducibles admiten activos por impuestos diferidos y generan un saldo a favor, que puede recuperarse a futuro (figura 5).
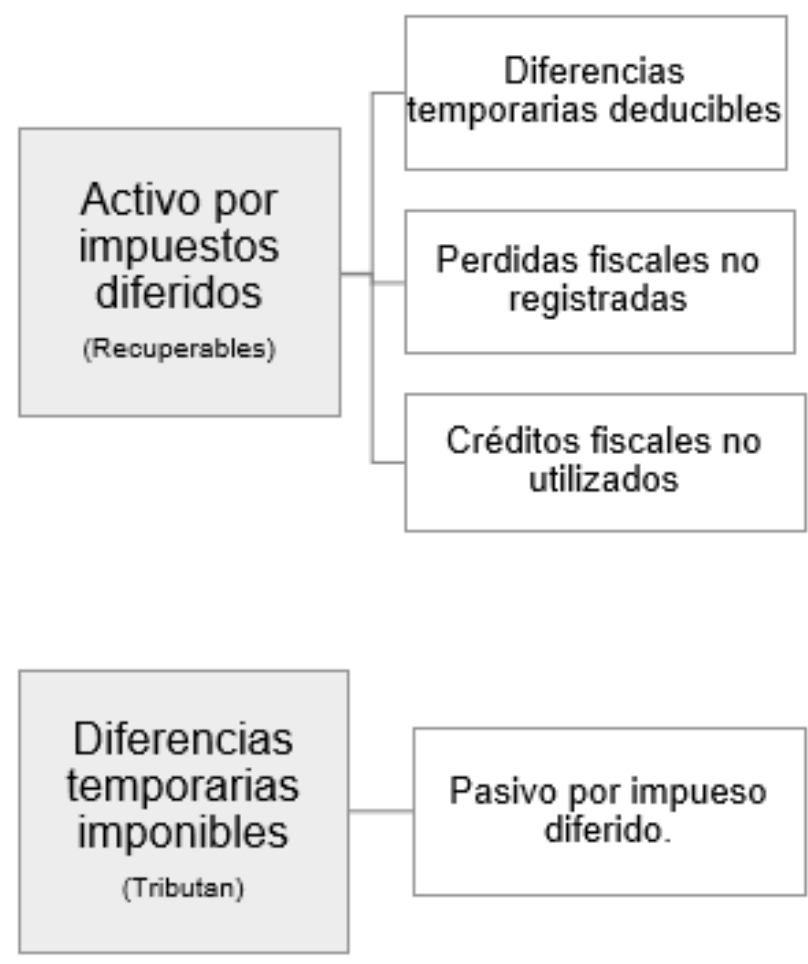

Figura 5. Diferencias temporarias.

Fuente: (Balseca, 2018)

b) Pasivos por impuestos diferidos: corresponde a valores a pagar en periodos futuros que guardan relación con las diferencias temporarias imponibles.

\section{Activo contable $>$ activo fiscal $=$ pasivo por impuesto diferido \\ Pasivo Contable < Pasivo Fiscal = pasivo por impuesto diferido}

Figura 6. Casos de pasivos por impuesto diferido.

Fuente: (Castro, 2016)

c) Activos por impuestos diferidos, son los recursos correspondientes a gastos anticipados como: intereses, seguros, arrendamientos y otros incurridos para recibir beneficios en los periodos posteriores

Los activos por impuestos diferidos están ligados a tres tipos de diferencias, en primer lugar las diferencias temporarias imponibles, denominadas de este modo pues generan cantidades imponibles al determinar la ganancia o pérdida fiscal correspondiente a periodos futuros cuando el importe en libros del activo sea recuperado o el pasivo sea liquidado.

\section{Activo contable $<$ activo fiscal $=$ activo por impuesto diferido \\ Pasivo contable $>$ pasivo fiscal $=$ activo por impuesto diferido}

Figura 7. Casos de activo por impuesto diferido.

Fuente: (Castro, 2016)

Por otro lado, están las diferencias temporarias deducibles al determinar la ganancia o pérdida fiscal correspondiente a períodos futuros, cuando el importe en libros del activo es recuperado o el pasivo sea liquidado, y por último las diferencias permanentes, que por su naturaleza, no afectan el impuesto a la renta, sino a las cuentas de gastos y no son factibles de compensación en periodos futuros (López \& Soto, 20191. 
Métodos de reconocimiento del impuesto diferido

La NIC 12, establece obligatoria la aplicación del método del pasivo basado en el balance general para realizar el proceso de cálculo de los impuestos diferidos. Consiste en comparar activos y pasivos, tanto financieros, como tributarios, y, de no estar en concordancia, genera una diferencia temporaria (IFRS Foundation, 2013).

El método del pasivo basado en el balance puede pasar por cuatro casos, dos de ellos generan un activo por impuesto a la renta diferido que son impuestos sobre las ganancias que pueden recuperarse en casos especiales. Y las otras dos generan un pasivo por impuesto a la renta diferido que son impuestos sobre las ganancias que deben pagarse en periodos futuros (Cavero \& Lozano, 2019).

Balseca (2018) indica que el procedimiento en el método del pasivo basado en el balance, es el siguiente:

\section{Fijar valores fiscales de activos y pasivos.}

2. Comparar valores contables de activos y pasivos con los fiscales y calcular las diferencias temporarias.

\section{Verificar que las diferencias temporarias no} sean excepciones dentro de la NIC 12.

4. Ajustar las diferencias temporarias (de inicio de año vs. las de fin de año)

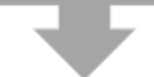

\section{Conciliar impuesto a las ganancias}

Figura 8. Método del pasivo, basado en el balance.

Fuente: Basado en (Balseca, 2018) (IFRS Foundation $\AA$, 2019)
Activos y pasivos de impuestos a la renta diferidos, vigentes en Ecuador

En junio del año 2012 el Servicio de Rentas Internas a través de una circular indicó que aquellos gastos que fueron considerados por los sujetos pasivos como no deducibles para efectos de la declaración del impuesto a la renta en un determinado periodo fiscal no serán deducibles en periodos posteriores, sin embargo, más adelante la Ley Orgánica de Incentivos a la Producción y Prevención del Fraude Fiscal, publicada en el año 2014 con registro Nro. 405, reformó la LORTI agregando al artículo 10 lo siguiente: "Impuestos diferidos.- Para efectos tributarios se permite el reconocimiento de activos y pasivos por impuestos diferidos, únicamente en los casos y condiciones que se establezcan en el reglamento" (Asamblea Nacional del Ecuador, 2014). Las condiciones aceptadas por la LORTI están contenidas en la figura 6.

Establecidos los lineamientos contables y tributarios, se debe realizar un análisis de los activos y pasivos por impuesto a la renta diferidos (figura 3).

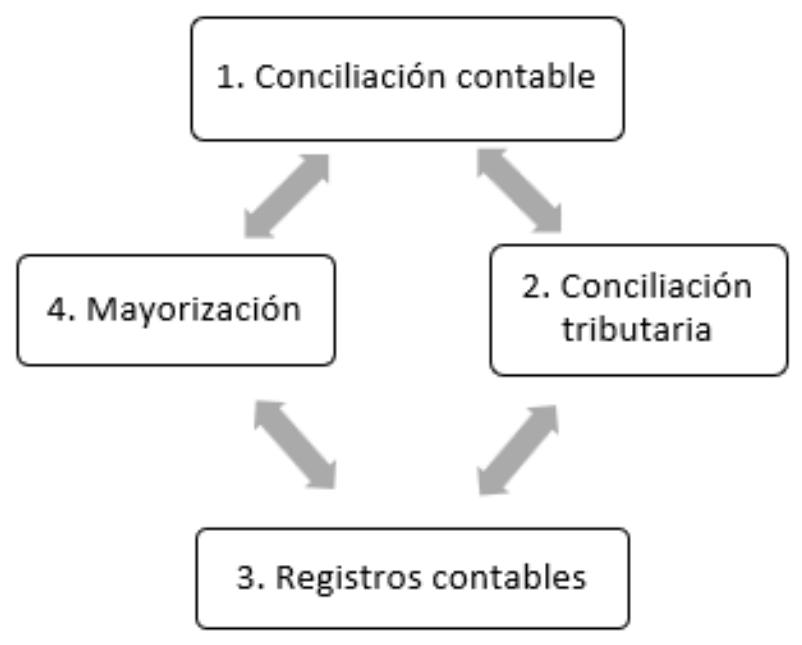

Figura 9. Análisis de Activos y pasivos.

Fuente: (Castro, 2016)

La conciliación contable registra el impuesto a la renta de la diferencia temporaria según corresponda a activo o a pasivo. 
En la conciliación tributaria se calcula la utilidad o pérdida del periodo fiscal vigente y se calcula el impuesto a la renta.

En el Registro contable se guardan las transacciones relacionadas al impuesto a la renta diferido.

En la Mayorización se cierra el periodo contable tomando en cuenta los valores diferidos /Castro, 2016).

Reconocimiento de impuestos diferidos en la Ley Orgánica de Régimen Tributario Interno

La Ley de Régimen Tributario Interno (LORTI), entró en vigencia desde el año 2004 y publicó su última reforma a finales del año 2015. La LORTI es la encargada de normar las declaraciones tributarias para lograr una recaudación de impuestos en base a lo establecido por ley, según sean personas naturales, compañías, sociedades nacionales y extranjeras; la administración de los tributos está a cargo del Servicio de Rentas Internas (SRI).

La LORTI reconoce los impuestos diferidos, sean estos activos o pasivos, con el requisito de realizar la prueba del VNR, es decir, cada ítem debe comparar su costo con su VNR y de ser menor, la perdida se verá reflejada en el estado de resultados. El impuesto diferido se generará para su deducción en el periodo fiscal siguiente.

La LORTI, reconoce impuestos diferidos, sean estos activos o pasivos, con restricción a los casos establecidos por el reglamento. Estos casos están contenidos en la figura 7.

\begin{tabular}{|l|}
\hline 1. Las pérdidas por deterioro de inventario. \\
\hline 2. Las pérdidas esperadas en contratos de construcción. \\
\hline 3. La depreciación. \\
\hline 4. Valor del deterioro de propiedades planta y equipo. \\
\hline 5. Cuentas incobrables y desmantelamientos. \\
\hline 6. Perdidas por mantención de inventario para la venta. \\
\hline 7. Activos biológicos. \\
\hline 8. Perdidas declaradas \\
\hline 9. Créditos tributarios no utilizados. \\
\hline 10. Contratos de servicios con financiamiento. \\
\hline 11. Provisiones para pagos por desahucio y jubilación patronal. \\
\hline 12. Procesos de fiscalización de avances de obra. \\
\hline
\end{tabular}

Figura 10: Casos en que se genera un impuesto diferido.

Fuente: (SRI, 2015)

El SRI establece que los impuestos diferidos deben estar debidamente soportados con información confiable, contenida en los estados financieros correspondientes a cada caso.

\section{Método}

El trabajo de investigación se desarrolló con una metodología mixta, por un lado cualitativa, pues se analizó el problema desde las perspectivas de la empresa y verificó el cumplimiento de la normativa tributaria y contable en los estados financieros sin manipular ninguna variable de estudio en la investigación. Por otro lado, es cuantitativa, en cuanto las herramientas utilizadas para la investigación permitieron establecer y evaluar la conciliación tributaria y su tratamiento contable relacionado a los impuestos diferidos.

Las técnicas de investigación empleadas fueron la encuesta y la entrevista, se realizó una encuesta al jefe departamental del área contable de la Agencia de Publicidad Ad/a. Adicional se aplicó una entrevista abierta con el administrador general de la agencia.

Los métodos de estudio utilizados fueron: histórico, al describir la temporalidad de las normativas utilizadas en el desarrollo de la propuesta, también el método analítico sintético que permitió analizar los componentes de las cuentas de impuestos diferidos para su correcta aplicación. 


\section{Resultados}

Se realizaron encuestas a los profesionales del departamento contable para conocer el nivel de conocimiento de las NIC 12. Los resultados evidenciaron la falta de conocimiento de la normativa y su proceso de aplicación. No se logró reconocer conocimientos expresos sobre la interpretación de los estados financieros con valores razonables, de vital importancia en el proceso de adopción de las NIIF en cualquier empresa. Otros resultados fueron:

Carencia del Manual de Funciones: Ios administradores consideran que, al tener solo dos personas en el departamento contable, no se hace necesaria la elaboración de un manual de funciones, las personas a cargo del departamento, han heredado el conocimiento mediante capacitaciones verbales o desde la experiencia, por lo que, el manual de funciones en el área de contabilidad, no existe, ni su creación está planeada para un futuro cercano.

Políticas Contables: las políticas contables son principios, reglas y procedimientos adoptados por una entidad para preparar y realizar los estados financieros. La investigación dio como resultado que las políticas contables dentro de la empresa no están establecidas mediante reglamento interno, sino que, las decisiones contables se encuentran supeditadas al criterio del contador general de la empresa.

Capacitación al personal sobre las normativas tributarias: la empresa no realiza capacitaciones al personal del departamento contable, pues no existe asignación presupuestaria para este rubro.

Confiabilidad en los estados financieros: Ios accionistas de la empresa no han presentado quejas en cuanto a la lectura de estados financieros, sin embargo, ha sido necesario el uso de un lenguaje entendible para facilitar la interpretación.

Aplicación de la NIC 12: la legislación ecuatoriana admite en ciertos casos los impuestos diferidos, por este motivo existen dudas entre la normativa contable y tributaria. El departamento contable tiene inconvenientes al contabilizar el impuesto sobre las ganancias, por la falta de conocimiento sobre las cuentas que deben usarse.

Diferencias temporarias y permanentes: las diferencias permanentes son aquellas que no pueden ingresarse como un gasto deducible; las diferencias temporarias son deducibles contable y fiscalmente cumpliendo algunos parámetros. La empresa no ha aplicado estos conceptos, por ende, tampoco la normativa en cuestión.

En la conciliación tributaria se registra impuestos diferidos: las empresas tienen que implementar un sistema de control fiscal para conciliar las diferencias que surgen entre los valores contables y fiscales, en la empresa no se han registrado los impuestos diferidos, pues estos valores incrementarían la utilidad del ejercicio para la recuperación en ejercicios posteriores, adicional, no existe el conocimiento para aplicar las contabilizaciones.

Estados financieros según NIIF: los estados financieros que la empresa ha presentado son: estado de situación financiera, estado de resultado integral, cambios en el patrimonio, flujos de efectivo y notas a los estados financieros, con lo que concluimos que la empresa presenta estados financieros según NIIF pero no aplica impuestos diferidos.

\section{Propuesta}

Mediante los resultados obtenidos en la investigación se determinó los inconvenientes al establecer las diferencias entre lo fiscal y contable dentro de la empresa Ad/a, por lo que se propone un procedimiento metodológico para aplicar la NIC 12 Impuesto a las Ganancias (figura 8) y así realizar la conciliación tributaria de una manera más rápida y eficaz. 


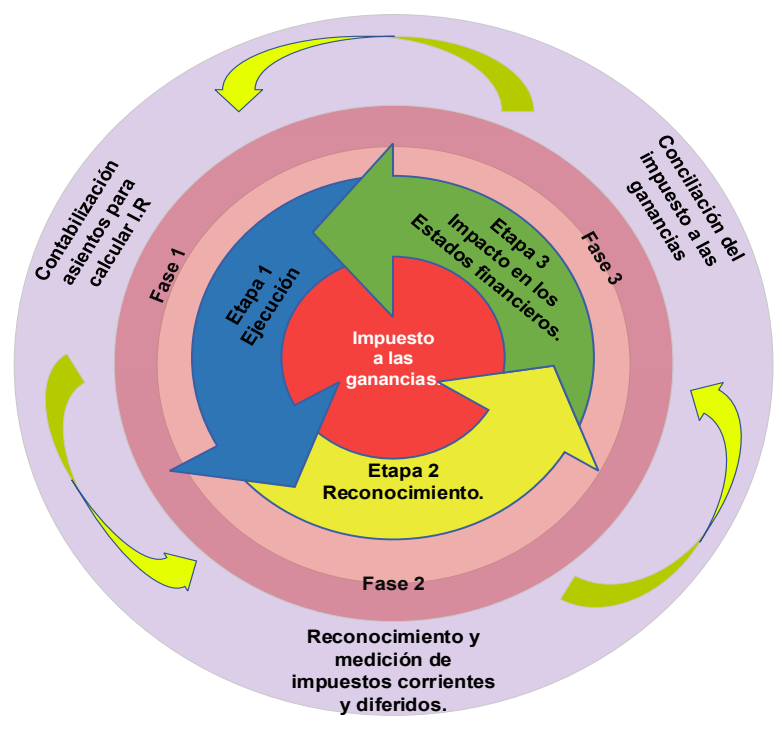

Figura 11. Procedimiento para la aplicación de la NIC 12 impuesto a las ganancias

\section{Etapa 1:}

Ejecución: Para la fase de ejecución se debe realizar un estudio previo de la normativa NIC 12, dado el impacto que provoca en el activo, pasivo, patrimonio e ingresos y gastos de la empresa, su aplicación hace obligatorio el análisis y evaluación de estos rubros para el reconocimiento, medición y determinación de la información a revelar.

Por ello, el balance de resultados debe estar disponible para la investigación, tomando en cuenta que los ingresos se miden en cuan gravables puedan llegar a ser y los gastos por su deducibilidad, después se obtiene la ganancia neta de un periodo determinado. En la tabla 1 la Agencia de Publicidad Ad/a obtiene el resultado contable.

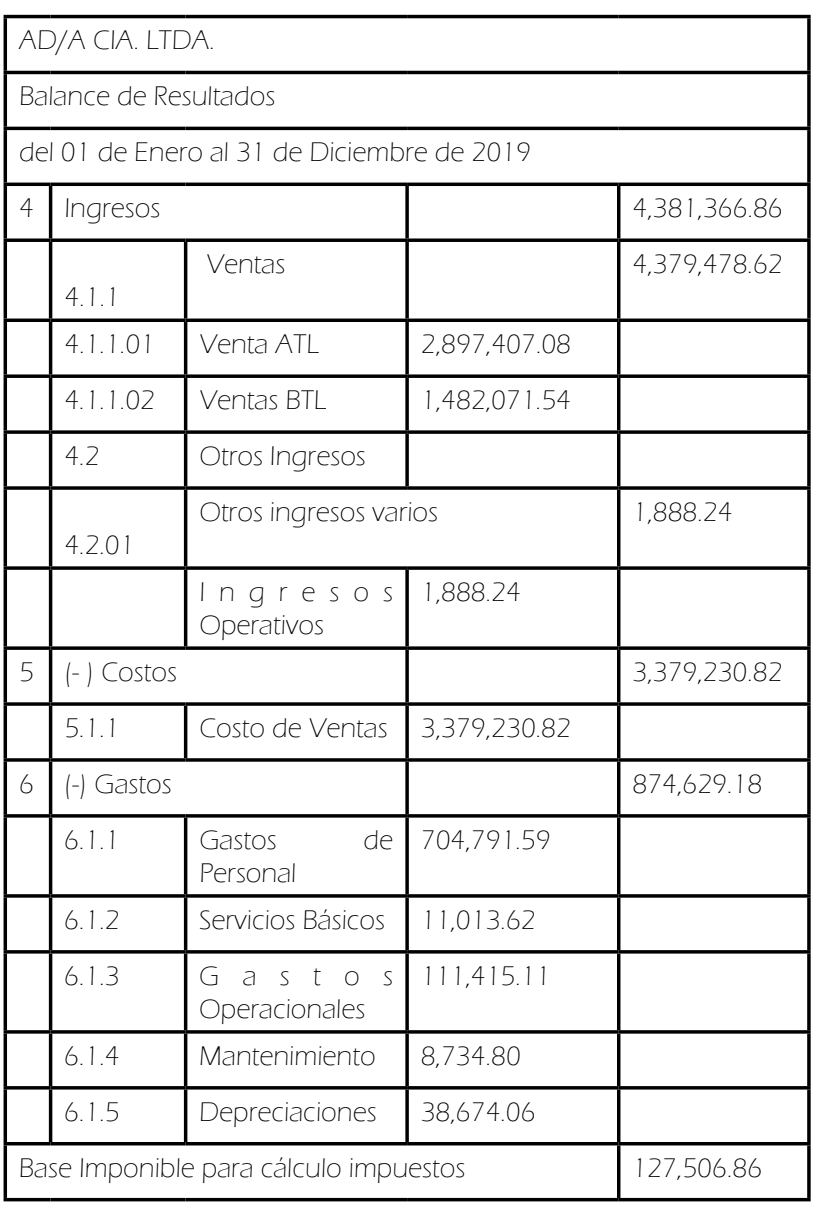

Tabla 1. Ingresos y gastos.

Fuente: Ad/a.

Los ingresos gravables de la empresa Ad/a son aquellos que están determinados en la ley de régimen tributario interno, obtenidos desde la prestación de servicios de publicidad, que es el giro del negocio, los gastos incurridos son deducibles ya que son indispensables para obtener los ingresos gravados.

\section{Etapa 2. Reconocimiento}

El impuesto corriente del periodo fiscal o de los anteriores debe ser reconocido como una obligación de pago de acuerdo como se haya liquidado. Según la NIC 12, en el caso ejemplo de las pensiones jubilares estos son pasivos que se acumulan hasta que se realicen los desembolsos y posterior sean deducibles en la conciliación tributaria (tabla 2).

Si el pago calculado excedió el importe a pagar, se reconoce como un activo a ser medido, y su recuperación se espera con la aplicación de 
la normativa y las tasas impositivas que están aprobadas a la fecha del pago.

Al cierre del ejercicio fiscal se analizarán los activos por impuestos diferidos que han sido reconocidos y pueden acceder a beneficios tributarios en ejercicios económicos posteriores. El reconocimiento de las diferencias existentes es visible en las cuentas de jubilación patronal y desahucio (tabla 2), cuyo estudio actuarial no se realizó en años anteriores por la falta de aplicación de la NIC 12.

\begin{tabular}{|l|l|l|}
\hline Cuenta & Valor & Diferencias \\
\hline $\begin{array}{l}\text { Gasto Prov. } \\
\text { Jubilación Patronal }\end{array}$ & $7,357.31$ & Temporario \\
\hline $\begin{array}{l}\text { Gasto Prov. } \\
\text { Desahucio }\end{array}$ & Temporario \\
\hline
\end{tabular}

Tabla 2. Reconocimiento de las diferencias existentes.

Fuente: Ad/a.

La jubilación patronal es un derecho del empleado y una obligación del empleador. En el Ecuador se encuentra regulada por el código de trabajo y por la LORTI en el Art. 10, literal 13, donde se indica que la totalidad de las provisiones de desahucio y jubilación patronal deben ser calculadas por un actuario e ingresadas en su totalidad siendo un gasto no deducible, salvo si el empleado presta sus servicios con el mismo empleador por más de diez años, en la tabla 3 se encuentra la contabilización de las provisiones de la empresa $\mathrm{Ad} /$ a.

\begin{tabular}{|l|l|l|}
\hline Detalle & Debe & Haber \\
\hline $\begin{array}{l}\text { Gasto Prov. De Jubilación } \\
\text { Patronal }\end{array}$ & $7,357.31$ & \\
\hline \multicolumn{2}{|c|}{ Cuentas x Pagar Jubilación Patronal } & $7,357.31$ \\
\hline $\begin{array}{l}\text { P/r provisión jubilación } \\
\text { patronal }\end{array}$ & & \\
\hline Gasto Prov. Desahucio & $4,507.40$ & \\
\hline $\begin{array}{l}\text { Cuentas x Pagar } \\
\text { Desahucio }\end{array}$ & & $4,507.40$ \\
\hline P/r provisión desahucio & & \\
\hline
\end{tabular}

Tabla 3 Contabilización de provisiones.

Fuente: Ad/a
Se reconocieron los gastos no deducibles de jubilación patronal y desahucio por ser empleados con menos de 10 años de servicio dentro de la empresa, situación que afecta directamente a la conciliación tributaria.

\section{Etapa 3.}

Impacto en los estados financieros

La NIC 12 permite realizar la medición de activos y pasivos diferidos de manera razonable.

\begin{tabular}{|l|l|}
\hline \multicolumn{2}{|l|}{ Agencia de Publicidad Ad/a } \\
\hline \multicolumn{2}{|l|}{ Conciliación Tributaria } \\
\hline (Expresado en U.S. dólares) & $127,506.86$ \\
\hline Utilidad Contable & $19,126.03$ \\
\hline (-) 15\% Participación de Trabajadores & 0.00 \\
\hline (-) Dividendos exentos & 0.00 \\
\hline (-) Otras rentas exentas & $4,507.40$ \\
\hline (+) Gastos no deducibles temporarios & $7,426.48$ \\
\hline (+) Gastos no deducibles permanentes & 0.00 \\
\hline (+) Gastos no deducibles del exterior & \\
\hline (+) Gastos incurridos para generar ingresos & 0.00 \\
\hline exentos y gastos atribuidos & \\
\hline (+) Participación trabajadores atribuible a & 0.00 \\
\hline ingresos exentos y no objeto impuesto renta & 0.00 \\
\hline (-) Deducciones adicionales & 0.00 \\
\hline (+) Ajustes por precios de transferencia & 0.00 \\
\hline (-) Ingresos sujetos a Impuesto a la Renta Único & $102,267.50$ \\
\hline (+) Costos y gastos deducibles incurridos para \\
\hline generar ingresos sujetos a IR. & $25,566.88$ \\
\hline Base Imponible para el impuesto a la renta & $120,314.71$ \\
\hline (-) 15\% de trabajadores & $3,835.03$ \\
\hline Utilidad después de trabajadores & $72,865.60$ \\
\hline (-) 25\% Impuesto a la Renta causado & $18,047.21$ \\
\hline Utilidad después de impuestos & \\
\hline (-) $5 \%$ Reserva Legal & \\
\hline Utilidad del Ejercicio & \\
\hline
\end{tabular}

Tabla 4. Conciliación Tributaria

La LORTI señala que los activos y pasivos por impuestos diferidos se miden a través de las tasas impositivas, en caso de la empresa Ad/a es el 25\%, en la Tabla 4 se observa la conciliación tributaria antes de los impuestos diferidos:

En la conciliación de las provisiones se generó un activo por impuestos diferidos, en la tabla 
5 se determina el impuesto generado, su valor puede ser recuperado en ejercicios posteriores.

\begin{tabular}{|l|l|}
\hline Provisión 2019 & \\
\hline Gasto Prov. De Jubilación Patronal & $7,357.31$ \\
\hline Gasto Prov. Desahucio & $4,507.40$ \\
\hline Total provisión 2019 & $11,864.71$ \\
\hline 25\% Impuesto a la Renta por & \\
\hline impuestos diferidos & $2,966.18$ \\
\hline
\end{tabular}

Tabla 5. Calculo impuesto diferido 2019.

Dado que las normas tributarias, son independientes de las contables, la conciliación fiscal, es el punto medio donde se establece la base sobre la que se calculará el impuesto a la renta, es también indispensable para ajustar la utilidad contable y tributaria que se reportará en el impuesto a la renta declarado al organismo de control estatal.

Se aplica la NIC 12 en la tabla 6, al generarse un activo por impuesto diferido, cumpliendo así la normativa.

\begin{tabular}{|l|l|}
\hline \multicolumn{2}{|l|}{ Agencia de Publicidad Ad/a } \\
\hline \multicolumn{2}{|l|}{ Conciliación Tributaria } \\
\hline (Expresado en U.S. dólares) & $127,506.86$ \\
\hline Utilidad Contable & $19,126.03$ \\
\hline (-) $15 \%$ Participación de Trabajadores & 0.00 \\
\hline (-) Dividendos exentos & 0.00 \\
\hline (-) Otras rentas exentas & $4,507.40$ \\
\hline (+) Gastos no deducibles temporarios & $7,426.48$ \\
\hline (+) Gastos no deducibles permanentes & 0.00 \\
\hline (+) Gastos no deducibles del exterior & 0.00 \\
\hline (+) Gastos incurridos para generar ingresos \\
\hline exentos y gastos atribuidos & \\
\hline (+) Participación trabajadores atribuible a & \\
\hline ingresos exentos y no objeto impuesto \\
renta
\end{tabular}

\begin{tabular}{|l|l|}
\hline (-) Deducciones adicionales & 0.00 \\
\hline (+) Ajustes por precios de transferencia & 0.00 \\
\hline $\begin{array}{l}\text { (-) Ingresos sujetos a Impuesto a la Renta } \\
\text { Único }\end{array}$ & 0.00 \\
\hline $\begin{array}{l}\text { (+) Costos y gastos deducibles } \\
\text { incurridos para generar }\end{array}$ & 0.00 \\
\hline ingresos sujetos a IR. & $120,314.71$ \\
\hline Utilidad Contable & $18,047.21$ \\
\hline (-) 15\% de trabajadores & $102,267.50$ \\
\hline Utilidad después de trabajadores & $25,566.88$ \\
\hline (-) 25\% Impuesto a la Renta causado & $76,700.63$ \\
\hline Utilidad después de impuestos & $3,835.03$ \\
\hline (-) 5\% Reserva Legal & $2,966.18$ \\
\hline (+) Impuestos diferidos & $75,831.78$ \\
\hline Utilidad del Ejercicio & \\
\hline
\end{tabular}

Tabla 6. Conciliación tributaria incluido impuestos diferidos.

En la tabla 7 la utilidad del ejercicio se incrementó en $\$ 2.966,18$ por lo que se generó un activo por impuesto diferido.

\begin{tabular}{|l|l|l|}
\hline Concepto & Debe & Haber \\
\hline Activo por I.R. diferido & $2,966.18$ & \\
\hline Ingresos por I.R. Diferido & $2,966.18$ \\
\hline $\mathrm{p} / \mathrm{r}$ Impuesto Diferido & & \\
\hline
\end{tabular}

Tabla 7. Contabilización impuestos diferidos.

El importe en libros de un activo por impuesto diferido debe revisarse al final de cada periodo para que la empresa disminuya el saldo del activo por impuesto diferido. En caso que no obtenga ganancia fiscal en períodos futuros, puede aplicarla contra la totalidad o una parte del valor. Para la reducción aplicada se debe realizar la reversión de acuerdo al valor recuperado, para finalmente dar de baja los saldos.

\section{Discusión}

En el Ecuador, a partir del año 2010 mediante resolución la Superintendencia de Compañías, 
Valores y seguros, adoptó las normas internacionales de información financiera, con la aplicación de la NIC 12 se contabilizan las partidas con efectos fiscales para conciliar lo tributario con lo contable, creando una cuenta de activo por impuesto diferido.

Los impuestos diferidos constituyen el valor a pagar o recuperar en periodos posteriores, y surgen como resultado de aplicar la normativa contable y tributaria; para efectos tributarios se puede aplicar el reconocimiento de activos y pasivos por impuestos diferidos a aquellos casos y condiciones establecidos en la norma tributaria. Las diferencias contablemente se aplican como activos o pasivos que se cobran o liquidan cuando se devengan como impuesto.

Las diferencias permanentes afectan a la utilidad contable puede ser al ingreso o al gasto, no generan impuestos diferidos debido a su naturaleza ya que no son compensables, por lo tanto, deben ser reconocidas en el periodo correspondiente afectando al impuesto corriente.

Las diferencias temporarias son los valores imponibles al determinar la ganancia o pérdida fiscal de periodos futuros ya que el importe en libros del activo se recuperará o el pasivo se liquidará, se aplican a través del método del pasivo basado en el balance.

Después de analizar la conciliación tributaria realizada por la empresa Ad/a, se detectó que el departamento contable de la empresa no aplica los impuestos diferidos por jubilación patronal y desahucio, afectando al estado de resultados y situación financiera; es decir, a las cuentas de activo, pasivo, patrimonio, ingresos y gastos, con la temida consecuencia de que los estados financieros presentados a los accionistas, no demuestran la situación real de la empresa, resultado de la falta de planificación tributaria, considerada por Encalada et al. (2020) como una herramienta para optimizar la carga fiscal.

El procedimiento propuesto para la conciliación de impuestos, su liquidación o recuperación, es factible de uso en periodos posteriores, su correcta contabilización determinará balances con razonabilidad e información real y comparable.

No se realizó un estudio comparativo de la aplicación de la normativa contable y tributaria, a falta de aplicación la NIC 12 Impuesto a las Ganancias en la empresa objeto de estudio.

\section{Referencias Bibliográficas}

Abascal, E. (2017). Fundamentos y técnicas de investigación comercial. España: Edibral.

Asamblea Nacional del Ecuador. (2014). Ley Orgánica de Incentivos a la Producción y Prevención del Fraude Fiscal,. Registro Oficial Suplemento 405, 19.

Balseca, N. (2018). Incidencia de la aplicación de impuestos diferidos en la presión tributaria a empresas. Creative Commons UASB Digital, 1-197.

Bernal, J. (2015). Origen y reversión de las diferencias temporarias y permanentes: Incidencia de la reducción de la tasa del impuesto a la renta - Aplicación de la NIC 12. Lima: Instituto Pacífico. Obtenido de ISBN: 9786124265211.

Cabrera, P., Narváez, C., \& Erazo, J. (2020). Análisis de la cultura tributaria de los consumidores y comerciantes de productos cárnicos en el cantón Santa Isabel, provincia del Azuay. Revista Científica Dominio de las Ciencias, 6(1). doi:https://dx.doi.org/10.23857/ dc.v6i1.1152

Castro, M. (2016). Activos y Pasivos Diferidos por concepto de Impuesto a la Renta, aceptados por la Ley de Régimen Tributario Interno. Universidad Andina simón Bolívar.

Cavero, C., \& Lozano, J. (2019). Impuesto a la renta diferido en el sector inmobiliario en los últimos 10 años. Una revisión de la literatura científica. UPNBOX. Obtenido de http://hdl.handle.net/1 1537/23032 
Cedeño, P. (2016). Aplicación de las Normas Internacionales de Información Financiera (NIIF) en el Ecuador. Revista Científica Dominio de las Ciencias. Obtenido de https://bit.ly/2AJUIFD

Cuenca, M., Gonzales, M., Higuerey, A., \& Villanueva, J. (2017). Adopción NIIF en Ecuador: Análisis pre y post sobre magnitudes contables de empresas cotizadas. X-Pedientes Económicos, 3041. Obtenido de https://Dit.ly/3hv6kfP

Encalada, D., Narvaez, C., \& Erazo, J. (2020). La planificación tributaria, una herramienta útil para la toma de decisiones en las comercializadoras de GLP. Revista Científica Dominio de las Ciencias. , 6(1). doi:https://dx.doi.org/10.23857/ dc.v6i1.1138

Espinoza, R. (2003). Teoría y práctica de los impuestos diferidos. México: Universidad Autónoma de Baja California.

Estupiñan, R. (2017). Estados Financieros NIC/ NIFF. Bogotá: Ecoe Ediciones.

Fierro, A. (2016). Contabilidad de pasivos con estándares internacionales para pymes. Bogotá: Ecoe Ediciones.

Filgueira, C. (2006). Manual Contable NIIF. Aplicación práctica. Chile: Ediciones Universidad Alberto Hurtado.

Freire, J. (2010). Guía de aplicación práctica sobre la implementación de las NIIF en el Ecuador. Quito.

Gay, J., \& Amat, O. (2008). Por la Senda de la Nueva Contabilidad, Analizando e Interpretando el NPGC. España: Ediciones Gestión 2000

Grajales, G., Vargas, C., \& Agudelo, L. (2019). Estatuto Tributario, Contextualización bajo IFRS (IASB). Bogotá: Ediciones de la U.
Guevara, M. (2018). Contabilidad de Instrumentos Financieros Renta Variable y Consolidación de Estados Financieros según NIIF. México: Instituto Mexicano de Contadores Públicos.

Hansen-Holm, J. (2011). NIIF Teoría y Práctica Manual para implementar las Normas Internacionales de Información Financiera (2 ed.). Guayaquil: Hansen-Holm \& Co.

Hansen-Holm, M. (2011). Manual de Obligaciones Tributarias. Guayaquil: Hansen-Hom \& Co. .

IASC - International Accounting Standards Committee. (2019). Norma Internacional de Contabilidad no 12 Impuesto a las Ganancias. 442 - 505. Obtenido de https://wnw.ifrs.org/

IFRS Foundation. (2013). NIC 2 Inventarios. Obtenido de https://Dit.ly/2U lym8v

IFRS Foundation @. (2019). Norma Internacional de Contabilidad no 12 Impuesto sobre las ganancias. Obtenido de ifrs.org

Instituto Mexicano de Contadores Públicos. (2015). Normas internacionales de información financiera (NIIF) : Parte A el marco Conceptual y los requerimientos. FRS Foundation Publicacions Department.

International Accounting Standards Board. (1979). Impuesto a las Ganancias NIC 12.

Jiménez, E. (2018). El marco conceptual del IASB y su contribución en la asimetría de información en el mercado crediticio. Revista Publicando, 5(15), 1279 - 1294. doi:ISSN 1390-9304

Juca, C. (2019). Estudio de la NIC 12 Impuestos Diferidos y el impacto con la Normativa Tributaria vigente en el Ecuador. UCE. doi:https://bit.ly/3hvWfA

López, T., \& Soto, F. (2019). Cómo revelan las sociedades anónimas abiertas chilenas los impuestos diferidos 
en sus estados financieros. Revista Activos, $\quad$ 17/1). doi:https://doi. org/10.15332/25005278.5396

Mesén, V. (2013). Aplicaciones prácticas de las NIC - Completas. Costa Rica: Tecnología de Costa Rica.

OlCE- Organización Interamericana de Ciencias Económicas. (2019). China ya ha convergido hacia los estándares internacionales IFRS \& ISA. Obtenido de NIC-NIIF: muw.ifrs.org

Omeñaca, J. (2009). Plan General de Contabilidad y PGC de Pymes. Barcelona: Ediciones Deusto.

Panchi, M. (2017). Una mirada del proceso de regulación contable internacional en el contexto de globalización. Revista de investigación SIGMA, 1(4). doi:http:// dx.doi.org/10.24133/sigma.v4i01.1138

Paredes, C., \& Deás, J. (s.f.). Aplicación de la Norma Internacional de Contabilidad n.o 12 «Impuesto diferido». Cofin habana. Obtenido de ISSN 2073-6061 RSPS 2139

Pérez, J., \& Fol, R. (2019). Impuestos diferidos (ISR) Determinación práctica de la aplicación NIIF. Costa Rica: Tax Editores.

Rincón, C., Flores, E., \& Narváez, J. (2018). Impuestos Diferidos. Bogotá: Ediciones de la U.

Rodríguez, M. (2004). Contabilización del Impuesto a las Ganancias: Método del Impuesto Diferido. Editor La Ley Editora e Impresora.

Ruíz, J., \& Garcimartín, C. (2007). Sistemas fiscales y procesos de desarrollo: elementos para el debate de América Latina. Madrir: Editorial Complutense.

Saldevila, P., Oliveras, E., \& Bagur, L. (2010). Contabilidad General con el Nuevo PGC. Barcelona: Profit Editorial.
Servicio de Rentas Internas. (2015). Ley Orgánica de Régimen Tributario Interno LORTI. Dirección Nacional Jurídica, 121.

Solar, M., \& Hidalgo, G. (201 1). Diferencias entre NIIF completas y la NIIF para las PYMES y su aplicación en las partidas contables. Comision del proyecto de aplicacion de las IFRS. Obtenido de https://bit.ly/3ewqlvo

SRI. (20 15). Ley Orgánica de Régimen Tributario. Registro Oficial Suplemento.

Supercias. (2010). Resolucion SC.ICI CPAIFRS.G.11.010 NIIF para PYMES.

Superintendencia de Compañias. (2006).

Superintendencia de Compañias. (2008). Resolución No. 08.G.DSC.010.

Ugalde, N. (2014). Las Normas Internacionales de Información Financiera: historia, impacto y nuevos retos de la IASB. Revista de Ciencias Económicas, 32-1.

Van-Horne, J., \& Wachowicz, J. (2002). Fundamentos de Administración Financiera. México: Pearson Educación.

Vásquez, N. (2013). Normas Internacionales de Información Financiera (NIIF - IFRS). Barcelona: Profit Editorial I.

Zeff, S. (2012). La evoluación del IASC al IASB y los retos que engrenta. Houston. 